\title{
A combined droplet train and ambient pressure photoemission spectrometer for the investigation of liquid/vapor interfaces
}

\author{
David E. Starr ${ }^{1, \#}$, Ed K. Wong ${ }^{2}$, Douglas R. Worsnop ${ }^{3}$, Kevin R. Wilson ${ }^{1}$, Hendrik Bluhm ${ }^{1, *}$ \\ ${ }^{1}$ Chemical Sciences Division, Lawrence Berkeley National Laboratory, Berkeley, CA 94720. \\ ${ }^{2}$ Molecular Foundry, Lawrence Berkeley National Laboratory, Berkeley, CA 94720. \\ ${ }^{3}$ Aerodyne Research Inc., Billerica , MA 01821.
}

\begin{abstract}
We describe a combined ambient pressure photoelectron spectroscopy/droplet train apparatus for investigating the nature and heterogeneous chemistry of liquid/vapor interfaces. In this instrument a liquid droplet train with typical droplet diameters from $50 \ldots 150 \mu \mathrm{m}$ is produced by a vibrating orifice aerosol generator (VOAG). The droplets are irradiated by soft X-rays $(100 \ldots 1500 \mathrm{eV})$ in front of the entrance aperture of a differentially pumped electrostatic lens system that transfers the emitted electrons into a conventional hemispherical electron analyzer. The photoemission experiments are performed at background pressures of up to several Torr, which allows the study of environmentally important liquid/vapor interfaces, in particular aqueous solutions, under equilibrium conditions. The exposure time of the droplet surface to the background gases prior to the XPS measurement can be varied, which will allow future kinetic measurements of gas uptake on liquid surfaces. As an example, a measurement of the surface composition of a $\chi=0.21$ aqueous methanol solution is presented. The concentration of methanol at the vapor/liquid interface is enhanced by a factor of about 3 over the bulk value, while the expected bulk value is recovered at depths larger than about $1.5 \mathrm{~nm}$.
\end{abstract}

\footnotetext{
\# Present address: Center for Functional Nanomaterials, Brookhaven National Laboratory, Upton, NY.

* Corresponding author. Email hbluhm@lbl.gov, phone (510) 4865431.
} 


\section{INTRODUCTION}

The properties of liquid/vapor interfaces strongly influence the abundance and reactivity of trace gas molecules that are important for many heterogeneous processes in atmospheric and environmental chemistry. In particular aqueous aerosols catalyze many heterogeneous reactions in the troposphere and can act as both sinks (for, e.g., $\mathrm{HNO}_{3}, \mathrm{HCl}, \mathrm{N}_{2} \mathrm{O}_{5}{ }^{1}$ ) and sources (e.g., halogen radicals ${ }^{2}$ ) for atmospheric trace gases. To date, little is known about the concentration of solution phase species at the liquid/vapor interface, which can significantly differ from the bulk solution concentration and is an important quantity in the modeling of heterogeneous reactions at liquid/vapor interfaces. ${ }^{3,4}$

Of particular importance in atmospheric and environmental science are aqueous solution interfaces. Under ambient conditions the vapor pressure of water or aqueous solutions is in the mTorr to Torr range (e.g., the equilibrium vapor pressure of water at its melting point is 4.58 Torr). A direct measurement of the liquid/vapor interface under ambient conditions is difficult due to the limited number of surface-sensitive experimental techniques that are chemically specific and can operate at elevated pressures in the Torr range. Scanning probe techniques ${ }^{5,6}$ as well as non-linear optical spectroscopies such as sum-frequency generation (SFG) and secondharmonic generation $(\mathrm{SHG})^{7,8}$ are capable of operating under non-Ultra-High Vacuum (UHV) conditions and have been used to study vapor/liquid interfaces. In general, however, it is difficult for these techniques to provide quantitative information about the chemical composition at the interface.

X-ray photoelectron spectroscopy (XPS) is a surface sensitive method that is elementspecific, quantitative and allows chemical identification of constituents in the surface region (e.g. their oxidation state). ${ }^{9}$ The application of XPS to the study of liquid/vapor interfaces is, however, not straightforward. Due to elastic and inelastic scattering of electrons by gas phase molecules at elevated pressures, the sample in conventional XPS instruments has to be kept under UHV conditions. The attenuation of the photoelectron signal in a gas environment is proportional to $\exp (-z \sigma p / k T)$, where $\sigma$ is the electron scattering cross section, and $z$ the distance that the electrons travel through a volume at pressure $p$. In order to perform XPS experiments at elevated pressures, the path length of the electrons through the gas has to be minimized. This can be 
achieved by moving the sample surface close to a differentially pumped aperture, behind which the pressure drops by several orders of magnitude. Once the electrons pass the plane of the aperture, scattering by gas molecules is greatly reduced. This approach has been used, beginning with Hans Siegbahn and coworkers early designs in the 1970's ${ }^{10,11,12}$, in all ambient pressure XPS instruments that have been designed to date. ${ }^{13,14,15,16,17,18,19,20,21}$ A similar approach has been taken in the application of meta-stable impact electron spectroscopy (MIES) $)^{22,23}$ for the study of surfaces, including liquids, at elevated pressures. ${ }^{24}$

In addition to the scattering of electrons by gas molecules, there are other obstacles to overcome in ambient pressure XPS instruments, namely that the X-ray source (synchrotron or anode) and the electron analyzer have to be kept under UHV. The X-ray source can be separated from the high pressure region either by differential pumping or by use of X-ray transparent windows, such as $\mathrm{Al}$ or $\mathrm{SiN}_{\mathrm{x}}$ membranes with thicknesses in the $100 \mathrm{~nm}$ range. The electron analyzer is kept under high vacuum using differential pumping between the sample cell and the analyzer. Depending on the aperture dimension (normally on the order of $0.1 \ldots 10 \mathrm{~mm}^{2}$ ), pumping speed and the type of gas pumped, typical pressure differentials across apertures vary from $10^{-4} \ldots 10^{-2}$, respectively. Therefore, for pressures in the Torr range in the sample cell (as needed for aqueous solutions) several differential pumping stages are required, which in turn reduce the solid angle of collection of electrons, depending on the aperture sizes and spacing. Traditionally, the effective pressure limit in ambient pressure XPS has been 1 Torr. The recent development of ambient pressure XPS instruments that use differentially-pumped electrostatic lens systems has overcome this limitation. These systems can operate at pressures above 5 Torr. ${ }^{19}$ There are three instruments based on this principle currently in operation, all at $3^{\text {rd }}$ generation synchrotron facilities (Advanced Light Source (ALS) beamline 9.3.2, see Ref. 19; ALS beamline 11.0.2, see Ref. 20; and at Bessy at the ISISS beamline, see Ref. 21). The experiments described here were done at beamline 11.0.2 at the ALS in Berkeley.

The investigation of liquid/vapor interfaces using surface science techniques is not only difficult because it necessitates working under non-UHV conditions due to the vapor pressure of the liquids, but also due to the challenge of preparing stable, clean liquid surfaces inside a vacuum chamber. For ambient pressure XPS and MIES experiments a number of different 
approaches have been developed over the last decades. H. Siegbahn and coworkers have used wires $^{12}$, rotating trundles ${ }^{25}$ and disks ${ }^{26}$ that are continuously moved through a liquid reservoir and are thereby coated by a thin liquid layer that is then investigated using XPS. Another approach is taken by Winter \& Faubel et al. who perform XPS on liquid microjets with jet diameters of below $10 \mu \mathrm{m} .{ }^{27,28}$ The jets are expanded into a measurement chamber with a working pressure of $10^{-5}$ Torr. Due to the low chamber pressure in these experiments measurements under realistic partial pressures of, e.g. water, are not possible. A similar technique was also used by Hans and Kai Siegbahn, yet with a larger aperture diameter $(0.7 \mathrm{~mm})$ at a flow rate of $1 \mathrm{ml} / \mathrm{min}^{10}$

The instrument that we describe here is a combination of ambient pressure XPS at pressures of up to 5 Torr and a vibrating orifice aerosol generator (VOAG) that produces a droplet train with droplets of stable size and spacing. Droplet trains have been used for some time in atmospheric science experiments to measure the uptake kinetics of gases by liquids. ${ }^{29,30}$ The advantage over the wire, trundle or rotating disk approach is that the droplet train (like the liquid jet) provides a continuously refreshed liquid surface from a reservoir that has not been in contact with the chamber atmosphere, preventing the accumulation of background gas contamination on the liquid surface. The advantage of droplet trains over liquid jets is that the droplets in the train are stable over long distances (up to $40 \mathrm{~cm}$ at $\mathrm{p}<60 \mathrm{Torr}^{30}$ and about a meter at $\mathrm{p}<20$ Torr $^{31}$ ), while liquid jets break up within a few centimeters after the aperture due to Raleigh instabilities in the liquid column. Droplet trains therefore allow one to vary the exposure time of the liquid surface to a surrounding gas over a much longer time scale than for a liquid jet. In addition, the background vapor pressure in a droplet train setup provides a means of controlling the temperature of the droplet surface, which will, after an equilibration time, match the temperature of a liquid surface in equilibrium with the vapor pressure of the background. A combined ambient pressure XPS/droplet train experiment is therefore well suited to examine the kinetics of chemical reactions at the liquid/vapor interface, which are of importance in atmospheric and environmental chemistry.

In the following we describe the experimental setup of a prototype of a combined ambient pressure XPS/droplet train experiment and discuss the main issues in the experiments. We will conclude by providing an example of our first XPS measurements on 0.21 mole fraction 
$(\chi=0.21)$ aqueous methanol droplets, addressing specifically the concentration enhancement of methanol at the solution/vapor interface.

\section{TECHNICAL DETAILS}

The instrument described here combines the synchrotron-based ambient pressure photoemission spectrometer at beamline 11.0.2 (see Ref. 20) at the ALS with a VOAG. A schematic of the setup is shown in Fig. 1. Liquid from an external reservoir is pumped by an HPLC pump ${ }^{32}$ at flow rates in the $\mathrm{ml} / \mathrm{min}$ range through $3.2 \mathrm{~mm}$ diameter Teflon tubing. After the pump the liquid passes through a $0.5 \mu \mathrm{m}$ particle filter ${ }^{33}$ to remove particulate impurities in the liquid, which could cause clogging of the orifice. The liquid is then forced through a stainless steel orifice $^{34}$ (30 to $75 \mu \mathrm{m}$ diameter) mounted on a piezoelectric element ${ }^{35}$. By oscillating the orifice using the piezoelectric element $(\sim 10 \mathrm{~V}$ peak-to-peak and $\sim 20-100 \mathrm{kHz})$ the liquid jet is broken up into regular droplets with a diameter that depends on both the flow rate and the driving frequency of the piezo. Similar experimental set-ups have been used in the past to generate liquid droplet trains. ${ }^{29,30}$ This train of droplets is positioned close to the entrance aperture of the electrostatic lens system of the ambient pressure XPS endstation, using a laser diode as an alignment guide that is directed down the optical axis of the electrostatic lens. Incident photons from beamline 11.0.2 irradiate the droplets in front of the aperture, and the emitted electrons are collected by the differentially-pumped lens system. A chilled liquid reservoir at the bottom of the chamber collects the droplets. The temperature of the collected liquid in the reservoir controls the background vapor pressure in the chamber.

The inset in Fig. 1 shows a close-up of the aperture region viewed from the top, i.e. parallel to the direction of the droplet train. Droplets pass the aperture at a distance of about two aperture diameters since at this distance the gas pressure (which drops rapidly across the plane of the aperture) has recovered to about $98 \%$ of the background pressure. ${ }^{19}$ Electrons are detected under the "magic angle" (54.7 deg) relative to the polarization of the incident photon beam which

simplifies quantitative analysis of the XPS spectra. ${ }^{36}$ Since the spot size of the incident X-ray beam at beamline 11.0.2 can be adjusted to the size of the droplets (about several tens of micrometers) and is well within the acceptance area of the electrostatic lens of the electron 
analyzer (on the order of the first aperture diameter, e.g. 100 to $300 \mu \mathrm{m}$ ), photoelectrons emitted from the droplet surface can be measured with high efficiency. The incident photon beam not only irradiates the droplet surface but also the gas in front of the droplets and in the gaps between them. Therefore, gas phase XPS peaks are also observed in the spectra. Since the binding energies of gas phase and condensed species differ from each other ${ }^{37}$, the surface peaks can in most cases readily be identified and analyzed from the XPS spectrum. Moreover, the composition of the gas phase can be determined from the gas phase XPS peaks at partial pressures above $\sim 0.05$ Torr, and allows calibration of sensitivity factors for quantitative XPS analysis.

Figure 2 shows a stroboscopic photograph of a typical droplet train (in this case prepared from a $40 \%$ ethanol solution) in front of the conically-shaped entrance aperture (diameter 0.3 $\mathrm{mm}$ ) of the differentially pumped lens system. The background vapor pressure is 4.3 Torr at a reservoir temperature of $-5.3{ }^{\circ} \mathrm{C}$. The orifice diameter $d_{o}$ of the VOAG is $50 \mu \mathrm{m}$. From the known flow rate $F(2.2 \mathrm{ml} / \mathrm{min})$, and the driving frequency $f$ of the piezo element $(54 \mathrm{kHz})$ the nominal droplet diameter $D_{d}$ is determined to be $110 \mu \mathrm{m}$ according to $D_{d}=(6 F / \pi f)^{1 / 3}$. $^{30}$ The droplet velocity $v_{d}$ is calculated from $v_{d}=4 F / \pi d_{o}^{2}$ and found to be $1870 \mathrm{~cm} / \mathrm{s} .{ }^{30}$ The typical distance between the orifice where the droplets are generated and the entrance aperture of the electrostatic lens system where the droplet surface is probed by XPS is $3 \mathrm{~cm}$ for the data shown in this paper. Since the droplets are at room temperature when they are generated, but the background vapor pressure in the measurement chamber (set by the reservoir temperature where the droplets are collected, see Fig. 1) is lower than the equilibrium vapor pressure of the solution at room temperature, the droplets initially evaporate rapidly until the equilibrium vapor pressure at their surface temperature matches the background vapor pressure in the chamber. The calculation of the droplet surface equilibration time is described in Ref. 30. Typical surface equilibration times under our conditions are about $0.5 \mathrm{~ms}$. Since the travel time of the droplets at $1870 \mathrm{~cm} / \mathrm{s}$ over a distance of $3 \mathrm{~cm}$ (between orifice and measurement position) is about $1.5 \mathrm{~ms}$, i.e. about a factor of three longer than the droplet equilibration time, we assume that under our conditions the droplets have equilibrated to the background vapor pressure when they reach the measurement position. No differences in the XPS spectra measured after a travel time of $1.5 \mathrm{~ms}$ 
(standard conditions) and $4.5 \mathrm{~ms}$ were observed, supporting our assumption that the droplet equilibration time is indeed less than $1.5 \mathrm{~ms}$. We will perform more experiments in the future to investigate this point in more detail.

The combination of droplet trains with ambient pressure XPS overcomes a number of problems that have hampered previous XPS experiments on liquid surfaces. The short time (milliseconds) between the generation of the droplets at the vibrating orifice and their measurement in the spectrometer minimizes the level of unwanted contamination on the surface. In addition, each droplet is irradiated only for a few microseconds by the incident X-rays thereby minimizing beam damage. For example, halides and organic molecules, which are important species in atmospheric and environmental science, are easily damaged by X-rays. ${ }^{38}$ Another advantage is that the interaction time of the droplet surface with gas molecules can be varied over a range from about 1 to $20 \mathrm{~ms}$, i.e. the reaction kinetics at liquid surfaces as a function of exposure time to a gaseous environment can be measured. In the setup described in this paper kinetic experiments are not straightforward, because the droplets and the reservoir are in contact with the background vapor and thus both can possibly react. Ideally, the droplet interaction region is isolated from both the droplet generation and droplet collection chambers, as described e.g. in Ref. 30. We are currently working on such a setup in our laboratory that will allow us to perform true kinetic measurements of gas uptake by liquid surfaces.

\section{PROOF OF PRINCIPLE EXPERIMENTS}

To test the feasibility of combined droplet train/photoemission experiments we have performed measurements on mixed methanol/water solutions. The goal of these experiments is to determine the composition of the methanol solution/vapor interface, in particular addressing the possibility of segregation of methanol to the vapor/solution interface, as suggested from Molecular Dynamics simulations ${ }^{39,40}$ and SFG measurements. ${ }^{41,42,43,44}$ Since the probing depth in XPS depends on the kinetic energy of the photoelectrons, which in turn is determined by the incident photon energy, depth-profiles of the stoichiometry at the solution/vapor interface can be measured, in the case described here up to a depth of about $2 \mathrm{~nm}$. 
Droplets were prepared from a solution with a methanol mole fraction of $\chi=0.21$ (corresponding to $37.5 \%$ methanol) using an orifice with a diameter of $50 \mu \mathrm{m}$, a flow rate of 2.2 $\mathrm{ml} / \mathrm{min}$ and an excitation frequency of $54 \mathrm{kHz}$, which yields a nominal droplet diameter of 110 $\mu \mathrm{m}$ and a droplet speed of $1870 \mathrm{~cm} / \mathrm{s}$ (see above). The distance between the VOAG and the measurement position was $3 \mathrm{~cm}$. The droplet reservoir was chilled to $-25{ }^{\circ} \mathrm{C}$, with a measured background vapor pressure in the chamber of 2.5 Torr. Figure 3 shows $\mathrm{C} 1 \mathrm{~s}$ and $\mathrm{O} 1 \mathrm{~s}$ photoemission spectra of the droplets. The $\mathrm{C} 1 \mathrm{~s}$ and $\mathrm{O} 1 \mathrm{~s}$ spectra were taken using incident photon energies of $730 \mathrm{eV}$ and $938 \mathrm{eV}$, respectively, i.e. the photoelectrons in those spectra had a similar kinetic energy of $\sim 450 \mathrm{eV}$. The combined analyzer/beamline resolution was about $0.5 \mathrm{eV}$. The position of the droplet train relative to the incident photon beam and the electron spectrometer was stable for several hours and allowed continuous measurement of photoemission spectra. Both gas phase and liquid phase photoemission peaks are visible. For a correct fit and subtraction of the gas phase signal from the XPS spectra the gas phase was measured separately by moving the droplet train away from the entrance nozzle of the differentially pumped electrostatic lens. The FWHM and relative binding energy differences from the pure gas phase peak fits were then kept constant in the fits of the combined liquid/vapor spectra.

The $\mathrm{C} 1 \mathrm{~s}$ peak in Fig. 3 of methanol in the solution is shifted by $\sim 1.0 \mathrm{eV}$ to lower binding energy (BE) from the gas phase methanol peak (note that the $\mathrm{C} 1 \mathrm{~s}$ gas phase peak is split due to vibrational excitations of the $\mathrm{C}-\mathrm{H}$ stretching mode ${ }^{45}$ ). In the O1s spectrum in Fig. 3 the methanol and water gas phase peaks are separated by $0.85 \mathrm{eV}$, in good agreement with literature values for the gas phase methanol and water BE difference. ${ }^{37}$ The liquid phase methanol and water peaks overlap too closely to be deconvolved in the fit.

Gas phase peak binding energies in the literature are normally referenced to the vacuum level instead of the Fermi level of a metal reference sample. The gas phase BEs shown in the spectra in Fig. 3 differ from those found in the literature by a factor related to the difference in the work function of the spectrometer $(4.6 \mathrm{eV})$ and of the surfaces that surround the measured gas volume, in our case the droplets and the entrance aperture of the electrostatic lens system (see inset in Fig. 1). The droplets are not in contact with ground, therefore they charge when irradiated by the beam, complicating the absolute binding energy calibration for solution peaks. 
The positive charging of the droplets is partially compensated by electrons that are generated through gas phase ionization when the incident photon beam passes though the chamber atmosphere (about $1.5 \mathrm{~cm}$ total path length before hitting the droplets). Droplet charging can be reduced by reducing the incident photon flux and is on the order of $+0.5 \mathrm{eV}$ in the spectra shown in Fig. 3.

From the fitted O1s methanol and water gas phase peak areas in Fig 3 we get a methanol/water ratio of 1.25 in the vapor, which corresponds to a mole fraction of methanol in the vapor of $\chi_{\text {vapor }} 0.56$. To our knowledge there are no previous measurements of the vapor and liquid mole fractions at $-25{ }^{\circ} \mathrm{C}$ for aqueous methanol solutions. Nevertheless, our experimental value of $\chi_{\text {vapor }}=0.56$ is consistent with $\chi_{\text {vapor }} \sim 0.6$ for a liquid mole fraction of 0.21 measured at 35 ${ }^{\circ} \mathrm{C} .{ }^{46}$ Due to the overlap in methanol and water liquid phase peaks the methanol/water ratio in the droplet cannot be directly determined from the O1s spectrum. Instead, this ratio is determined by the following method. First, the contribution of methanol to the O1s liquid phase peak is calculated by measuring the $\mathrm{C} 1 \mathrm{~s}$ methanol liquid phase peak and calculating its corresponding $\mathrm{O} 1 \mathrm{~s}$ liquid phase intensity using an experimentally-determined $\mathrm{C} 1 \mathrm{~s} / \mathrm{O} 1 \mathrm{~s}$ sensitivity factor for each $\mathrm{KE}$. This factor is obtained from the relative methanol $\mathrm{C} 1 \mathrm{~s}$ and $\mathrm{O} 1 \mathrm{~s}$ gas phase peak intensities measured at the kinetic energies used to depth profile the solution/vapor interface. Using these sensitivity factors, a depth-dependent methanol/water stoichiometry was calculated which is plotted in Fig. 4. The dotted line in Fig. 4 marks the bulk molecular ratio of methanol/water (0.26) from the bulk concentration of a $\chi_{\text {liquid }}=0.21$ methanol/water solution. At high kinetic energies (i.e. greater probing depth) the expected bulk value of methanol/water $=0.26$ is approached, while the most surface-sensitive measurements show a clear enhancement in the methanol-to-water ratio of about a factor of 3 over the bulk value. This factor of 3 enhancement represents a lower limit for the concentration of methanol directly at the vapor/solution interface since XPS integrates from the surface into the bulk up to a depth that is determined mainly by the inelastic mean free path (IMFP) of the electrons in the material as well as the detection geometry (see below). The error bars in Fig. 4 arise from uncertainties in the fitting of the C1s spectra (the uncertainties in the fitted O1s spectra are much smaller due to the higher signal-to-noise ratio there). The error bars represent the standard deviation in the methanol/water ratio that arises from 
using either a $0.9 \mathrm{eV}$ or $1.0 \mathrm{eV}$ binding energy difference between the liquid and gas phase $(v=0)$ methanol peaks (the range of binding energy differences that was found in unconstrained fits of the $\mathrm{C} 1 \mathrm{~s}$ spectra at the various kinetic energies), and also from uncertainties in the background subtraction.

In order to compare these data with the results of molecular dynamics (MD) simulations ${ }^{39}$ we need to determine the probing depth in our experiments as a function of electron kinetic energy. Because the IMFP of electrons in aqueous solutions is not well known we calculated the IMFP using the model by Tanuma, Powell and Penn ${ }^{47}$ for our experimental conditions, under consideration of the take-off angle of the electrons in our detection geometry. Since the spherical droplets are constantly moving through the field-of-view of the spectrometer, this geometric factor is not constant. Thus we approximate the droplet beam as a planar sample where the detection angle relative to the surface normal is 40 deg, i.e. the IMFP values are multiplied by a factor of $\cos (40 \mathrm{deg}) \sim 0.77$ to get an estimate of the probing depth. The calculated IMFP values as a function of kinetic energy are shown in Fig. 4.

For a qualitative comparison of the experimental results shown in Fig. 4 with the results of the MD simulations we have integrated the data in Fig. 1 of Ref. 39 over an the interfacial region of $1 \mathrm{~nm}$ from the liquid/vapor interface, which is comparable to the minimum probing depth in our experiments (at a kinetic energy of $200 \mathrm{eV}$ ). The MD simulations predict an enhancement of a factor of $\sim 2$ for a $\chi=0.5$, and a factor of $\sim 8$ for a $\chi=0.1$ solution, respectively. Our experimentally-determined surface enhancement of $\sim 3$ for the $\chi=0.21$ is in qualitative agreement with the MD simulations.

The results presented in Fig. 4 can be used to estimate the surface tension of the methanol water solution. The surface tension of a binary mixture $(\Gamma)$ can be approximated by a linear combination of the surface tensions of the pure components, ${ }^{48}$ weighted by their surface mole fractions $(\chi)$. In our case, $\Gamma=\chi_{\text {surf,meth }} \Gamma_{\text {meth }}+\chi_{\text {surf,water }} \Gamma_{\text {water }}$ where a $\mathrm{CH}_{3} \mathrm{OH} / \mathrm{H}_{2} \mathrm{O}$ ratio of 0.6 , observed in Fig. 4, corresponds to $\chi_{\text {surf,meth }}=0.37$ and $\chi_{\text {surf,water }}=0.63$. Using the surface tension of the pure components at $20{ }^{\circ} \mathrm{C}$ (water, $73.0 \mathrm{mN} / \mathrm{m}$ and methanol, $22.6 \mathrm{mN} / \mathrm{m}^{49}$ ), we estimate that the surface tension of the binary solution is $54 \mathrm{mN} / \mathrm{m}\left(\chi_{\text {bulk, meth }}=0.21\right)$. This value is larger than those reported in Refs. 49 and $50\left(\sim 40 \mathrm{mN} / \mathrm{m}\right.$ at $\left.20{ }^{\circ} \mathrm{C}\right)$, and Ref. $51\left(\sim 44 \mathrm{mN} / \mathrm{m}\right.$ at $\left.-10{ }^{\circ} \mathrm{C}\right)$ for 
solutions of the same composition. The reasons for this discrepancy are unclear. On the experimental side this could be due to an underestimate of the true surface concentration of methanol, since even at the lowest electron kinetic energies our signal still originates from a depth on the order of 2-3 molecular diameters $(\sim 8 \AA)$, as seen in Fig. 4 . The discrepancy could also be due to deviations from the ideal linear dependence of the surface tension on the surface mole fraction of methanol, which we have assumed in our calculations.

\section{CONCLUSIONS}

A combined droplet train/ ambient pressure photoelectron spectroscopy instrument for the investigation of liquid/vapor interfaces at background pressures of up to several Torr was designed and tested. This instrument offers the opportunity to study a wide range of liquids in a controlled gas environment, which are of particular interest for the investigation of liquid/vapor interfaces with relevance in atmospheric and environmental chemistry. The utility of the new technique is illustrated by the experiments on $\chi=0.21$ methanol/water solution that show methanol segregation at the solution/vapor interface. Our measurements allow us to estimate a lower limit for the surface enhancement of methanol for a $\chi=0.21$ methanol/water solution of a factor of $\sim 3$, and a maximum thickness for the interfacial region of $1.5 \mathrm{~nm}$, which is in qualitative agreement with the results of previous MD simulations.

\section{ACKNOWLEDGEMENTS}

We would like to thank Erin Mysak (Lawrence Berkeley National Laboratory, Berkeley, CA), Maria Krisch, Matthew A Brown and John C. Hemminger (University of California at Irvine, CA) for helpful discussions and assistance. This work is supported by a Laboratory Directed Research and Development project through Lawrence Berkeley National Laboratory. The ALS and the MES beamline 11.0.2 are supported by the Director, Office of Science, Office of Basic Energy Sciences, Division of Chemical Sciences, Geosciences, and Biosciences and Materials Sciences Division of the U.S. Department of Energy at the Lawrence Berkeley National Laboratory under Contract No. DE-AC02-05CH11231. 


\section{FIGURE CAPTIONS}

Figure 1: Schematic drawing of the combined ambient pressure photoemission/droplet train setup. Liquid from an external reservoir is pumped through an orifice (diameter 30 to $75 \mu \mathrm{m})$. The orifice is vibrated with frequencies in the 20 to $100 \mathrm{kHz}$ range, which leads to the formation of evenly sized and spaced droplets that traverse the vacuum chamber. The droplets are collected at the bottom of the chamber in a temperaturecontrolled reservoir. Incident photons irradiate the droplets and gas phase in the space between the droplets in front of the entrance aperture of a differentiallypumped electrostatic lens system that transfers the photoelectrons to the entrance aperture of a hemispherical analyzer. The inset shows a close-up of the droplet train in front of the entrance aperture of the lens system, viewed along the droplet propagation direction.

Figure 2: Stroboscopic photograph of a droplet train prepared from a $40 \%$ ethanol solution in front of the entrance aperture (diameter $0.3 \mathrm{~mm}$ ) of the differentially pumped lens system. The parameters are: background vapor pressure 4.3 Torr, orifice diameter of the VOAG $50 \mu \mathrm{m}$, flow rate $2.2 \mathrm{ml} / \mathrm{min}$, driving frequency of the piezo element 54 $\mathrm{kHz}$, nominal droplet diameter $D_{d} 110 \mu \mathrm{m}$, nominal droplet velocity $1870 \mathrm{~cm} / \mathrm{s}$.

Figure 3: C1s and O1s XPS spectra of methanol solution droplets (methanol mole fraction 0.21). The droplet parameters are similar to those of the ethanol solution droplets shown in Fig. 2. The $\mathrm{C} 1 \mathrm{~s}$ and $\mathrm{O} 1 \mathrm{~s}$ spectra were taken using incident photon energies of $730 \mathrm{eV}$ and $938 \mathrm{eV}$, respectively, i.e. at a photoelectron kinetic energy of $\sim 450 \mathrm{eV}$. Both gas phase and liquid phase photoemission peaks are visible in the $\mathrm{C} 1 \mathrm{~s}$ and $\mathrm{O} 1 \mathrm{~s}$ spectra.

Figure 4: Methanol/water ratio as a function of photoelectron kinetic energy. At the highest kinetic energies the expected bulk methanol/water ratio for a $\chi_{\text {liquid }}=0.21$ solution is recovered, while for low kinetic energies the ratio is enhanced in favor of methanol, indicating segregation of methanol to the solution/vapor interface. For details see text. 


\section{References}

1 J.M. Van Doren, L.R. Watson, P. Davidovits, D.R. Worsnop, M.S. Zahniser, C.E. Kolb, J. Phys. Chem., 1990, 94, 3265.

2 E. Knipping, M.J. Lakin, K.L. Foster, P. Jungwirth, D.J. Tobias, R.B. Gerber, D.Dabdub, B. Finlayson-Pitts, Science, 2000, 288, 301.

3 P. Jungwirth and D. Tobias, J. Phys. Chem. B, 2002, 106, 6361.

4 S. Ghosal, J.C. Hemminger, H. Bluhm, B.S. Mun, E.L.D. Hebenstreit, G. Ketteler, D.F. Ogletree, F.G. Requejo, M. Salmeron, Science, 2005, 307, 563.

5 A. Döppenschmidt, M. Kappl, H.-J. Butt, J. Phys. Chem. B, 1998, 102, 7813.

6 H. Bluhm, M. Salmeron, J. Chem. Phys., 1999, 111, 6947.

7 Y.R. Shen,V. Ostroverkhov, Chem. Rev., 2006, 106, 1140.

8 X. Wei, P.B. Miranda, Y.R. Shen, Phys. Rev. Lett., 2001, 86, 1554.

9 S. Huefner, Photoelectron Spectroscopy, Springer, Berlin, 1995.

10 H. Siegbahn, K. Siegbahn, J. Electron Spectrosc. Rel. Phenom., 1973, 2, 319.

11 H. Siegbahn, J. Phys. Chem., 1985, 89, 897.

12 H. Fellner-Feldegg, H. Siegbahn, L. Asplund, P. Kefve, K. Siegbahn, J. Electron Spectrosc. Rel. Phenom., 1975, 7, 421.

13 R.W. Joyner, M.W. Roberts, K. Yates, Surf. Sci., 1979, 87, 501.

14 K. von Burg, P. Delehay, Chem. Phys. Lett., 1981, 78, 287.

15 R.E. Ballard, R.E. Jones, D. Read, Chem. Phys. Lett., 1985, 121, 45.

16 M.A. Kelly. M.L. Shek, P. Pianetta, T.M. Gür, M.R. Beasley, J. Vac. Sci. Technol. A, 2001, 19, 2127.

17 H.-J. Ruppender, M. Grunze, C.W. Kong, M. Wilmers, Surf. Interface. Anal., 1990, 15, 245.

18 J. Pantförder, S. Pöllmann, J. F. Zhu, D. Borgmann, R. Denecke, H.-P. Steinrück, Rev. Sci. Instrum., 2005, 76, 014102. 
19 D.F. Ogletree, H. Bluhm, G. Lebedev, C.S. Fadley, Z. Hussain, M. Salmeron, Rev. Sci. Instrum., 2002, 73, 3872.

20 H. Bluhm, K. Andersson, T. Araki, K. Benzerara, G.E. Brown, J.J. Dynes, S. Ghosal, M.K. Gilles, H.-Ch. Hansen, J.C. Hemminger, A.P. Hitchcock, G. Ketteler, A.L.D. Kilcoyne, E. Kneedler, J.R. Lawrence, G.G. Leppard, J. Majzlam, B.S. Mun, S.C.B. Myneni, A. Nilsson, H. Ogasawara, D.F. Ogletree, K. Pecher, M. Salmeron, D.K. Shuh, B. Tonner, T. Tyliszczak, T. Warwick, T.H. Yoon, J. Electron Spectrosc. Relat. Phenom., 2006, 150, 86.

21 H. Bluhm, M. Haevecker, K. Ihmann, E. Kleimenov, D. Teschner, D.F. Ogletree, M. Salmeron, A. Knop-Gericke, R. Schloegl, in preparation.

22 Y. Harada, S. Masuda, H. Ozaki, Chem. Rev., 1997, 97, 1897.

23 H. Morgner. Adv. At. Molec. Opt. Phys., 2000, 42, 387.

24 W. Keller, H. Morgner, W.A. Müller, Molec. Phys., 1986, 57, 623.

25 H. Siegbahn, S. Svensson, M. Lundholm, J. Electron Spectrosc. Relat. Phenom., 1981, 24, 205.

26 R. Moberg, F. Bökman, O. Bohman, H. Siegbahn, J. Am. Chem. Soc., 1991, 113, 3663.

27 B. Winter, R. Weber, W. Widdra, M. Dittmar, M. Faubel, I.V. Hertel, J. Phys. Chem. A, 2004, 108, 2625.

28 B. Winter, R. Weber, I.V. Hertel, M. Faubel, P. Jungwirth, E.C. Brown, S.E. Bradforth, J. Am. Chem. Soc., 2005, 127, 7203.

29 J.A. Gardner, L.R. Watson, Y.G. Adewuyi, P. Davidovits, M.S. Zahniser, D.R. Worsnop, C.E. Kolb, J. Geophys. Res., 1987, 92, 10887.

30 D.R. Worsnop, M.S. Zahniser, C.E. Kolb, J.A. Gardner, L.R. Watson, J.M. Van Doren, J.T. Jayne, P. Davidovits, J. Phys. Chem., 1989, 93, 1159.

31 D.R. Worsnop, personal communication.

32 Dynamax Solvent Delivery System SD-200, Rainin Instrument Company, Inc., Woburn, MA. 
33 Swagelok Co., Solon, OH.

34 O'Keefe Controls Co., Trumbull, CT.

35 Morgan Electro Ceramics Inc., Bedford, OH; PZT8 Ring, OD 38 mm, ID 15 mm, thickness $6.3 \mathrm{~mm}$.

36 See, e.g., J.J. Yeh, I. Lindau, Atomic Data and Nuclear Data Tables, 1985, 32, 1.

37 See, e.g., K. Siegbahn, C. Nordling, G. Johansson, J. Hedman, P.F. Hedén, K. Hamrin, U. Gelius, T. Bergmark, L.O. Werme, R. Manne, Y. Baer, ESCA Applied to Free Molecules, North-Holland, Amsterdam, 1969, pp. 56-60.

38 See, e.g., J.H. Thomas III, in: A.W. Czanderna, C.J. Powell, T.E. Madey (Eds.), Beam Effects, Surface Topography, and Depth Profiling in Surface Analysis, Springer, Berlin,1999, pp.1.

39 T.-M. Chang, L.X. Dang, J. Phys. Chem. B, 2005, 109, 5759.

40 L. Pártay, P. Jedlovszky, Á. Vincze, G. Horvai, J. Phys. Chem. B, 2005, 109, 20493.

41 R. Superfine, J.Y. Huang, Y.R. Shen, Phys. Rev. Lett., 1991, 66, 1066.

42 C.D. Stanners, Q. Du, R.P. Chin, P. Cremer, G.A. Somorjai, Y.R. Shen, Chem. Phys. Lett., 1995, 232, 407.

43 K. Wolfrum, H. Graener, A. Laubereau, Chem. Phys. Lett., 1993, 213, 41.

44 H. Chen, W. Gan, R. Lu, Y. Guo, H. Wang, J. Phys. Chem. B, 2005, 109, 8064.

45 M. Wiklund, A. Jaworowski, F. Strisland, A. Beutler, A. Sandell, R. Nyholm, S.L. Sorensen, J.N. Andersen, Surf. Sci., 1998, 408, 210.

46 M.L. McGlashan, A.G. Williamson, J. Chem. Engineer. Data, 1976, 21, 196.

47 S. Tanuma, C.J. Powell, D.R. Penn, Surf. Interface Anal., 1988, 11, 577; we have calculated the IMFP using the NIST IMFP database (\#69), assuming a band gap for water of $6.9 \mathrm{eV}$.

48 J.G. Eberhart, J. Phys. Chem, 1966, 70, 1183.

49 CRC Handbook of Chemistry and Physics, $60^{\text {th }}$ Edition, CRC Press, New York, 1979. 
50 A. Ozkan, J. Colloid Interface Sci., 2004, 277, 437.

51 Q. Dong, K.N. Marsh, B.E. Gammon, A.K.R. Dewan (Eds.), Transport Properties and related thermodynamic data of binary mixtures, Part 3, Design Institute for Physical Property Data, New York, 1996, p. 298. 
Fig. 1

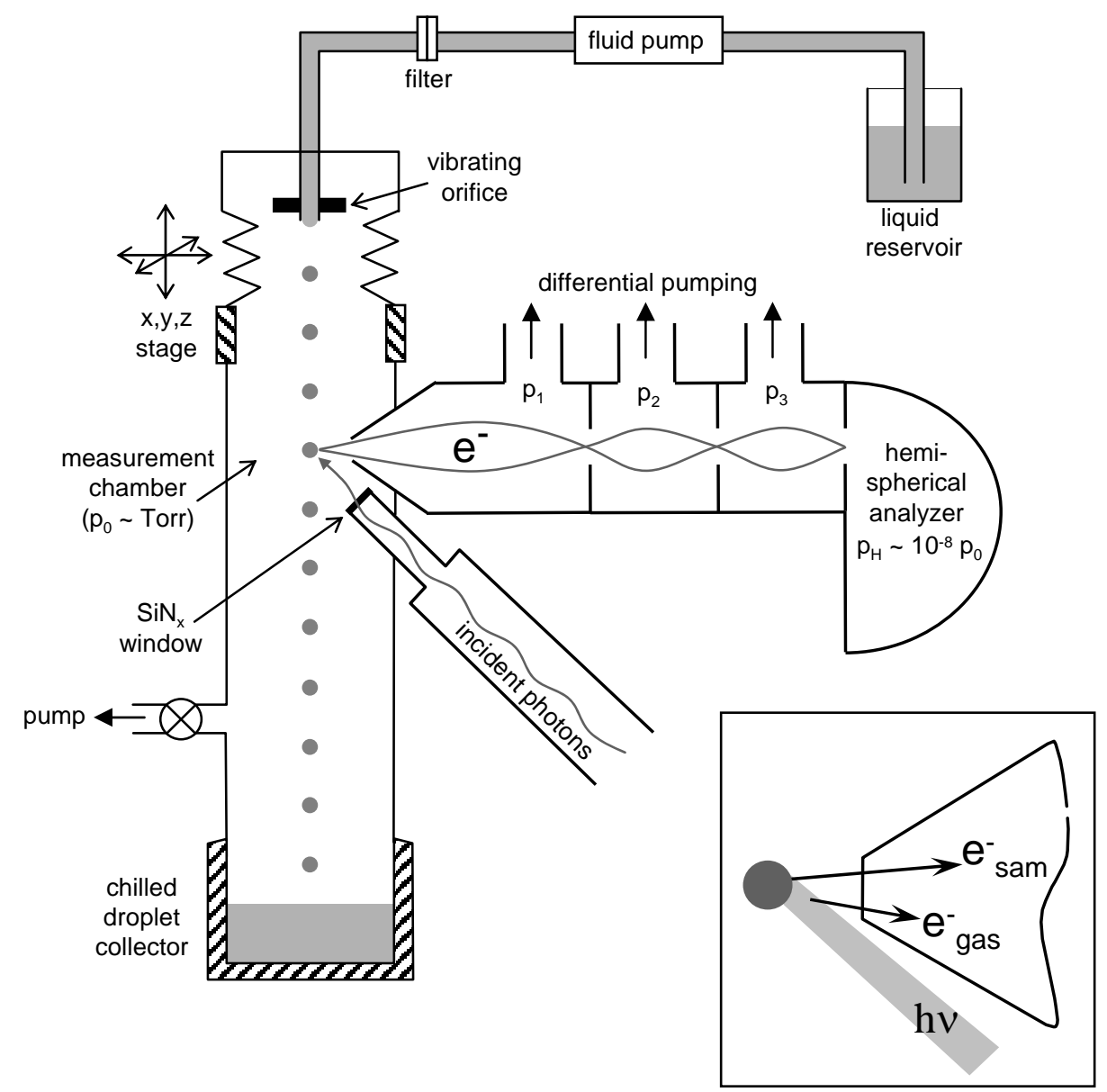


Fig. 2

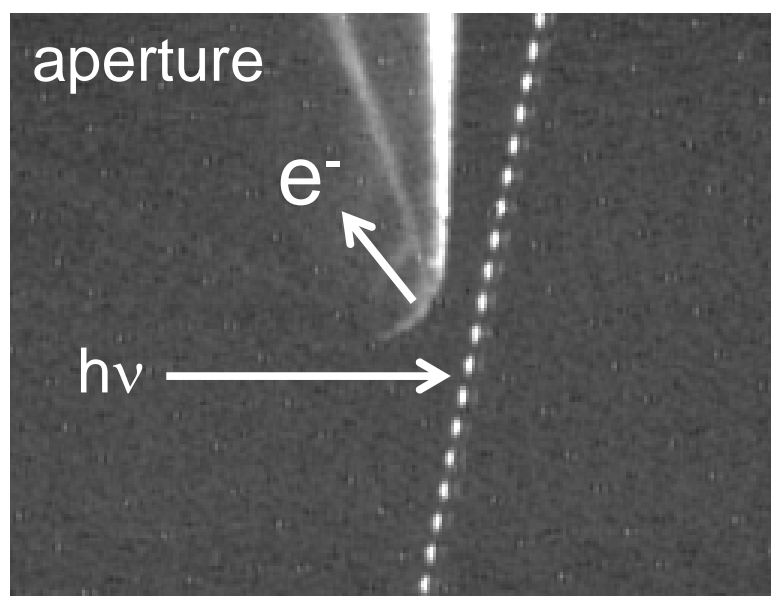

D.E. Starr, E.K. Wong, D.R. Worsnop, K.R. Wilson, H. Bluhm 
Fig. 3
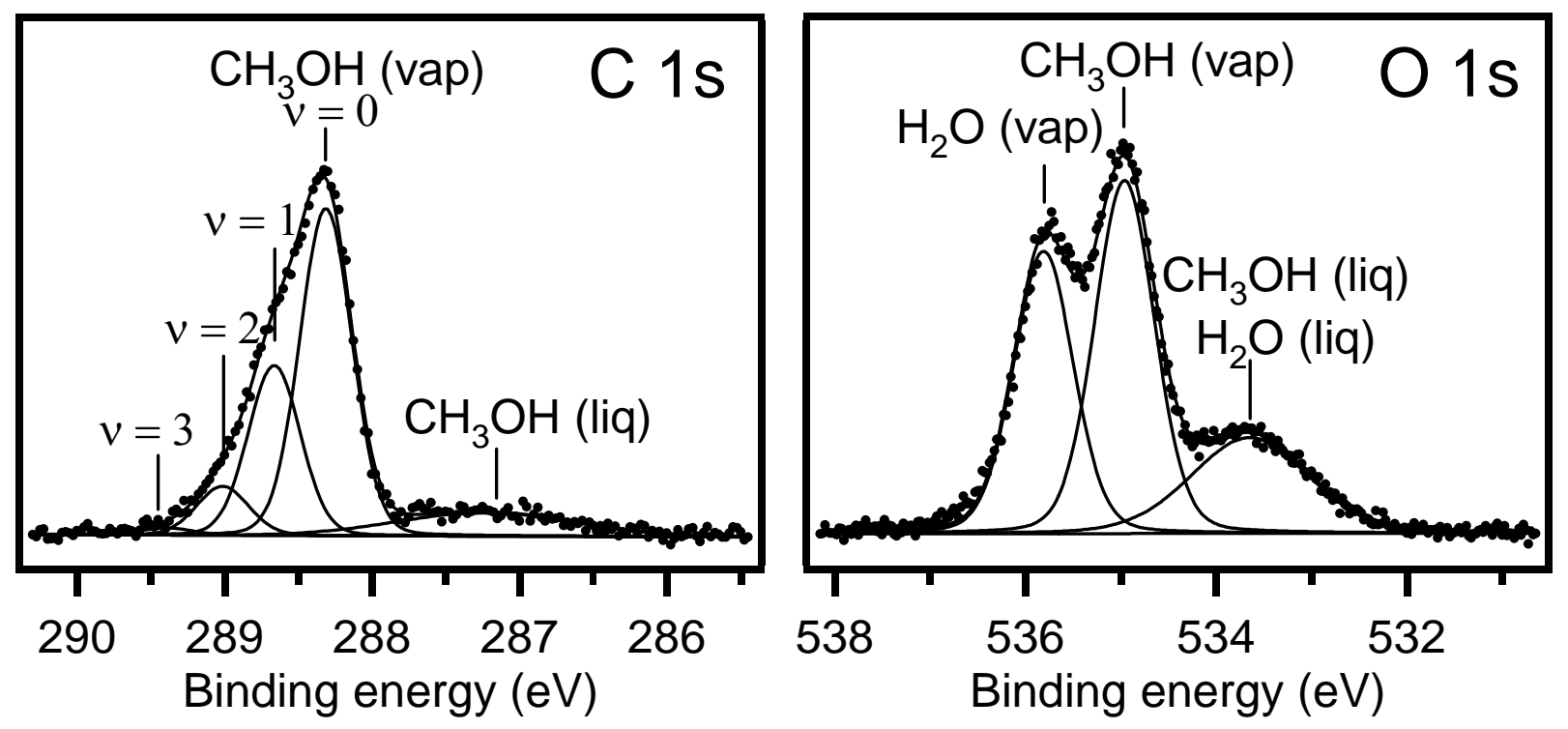

D.E. Starr, E.K. Wong, D.R. Worsnop, K.R. Wilson, H. Bluhm 
Fig. 4

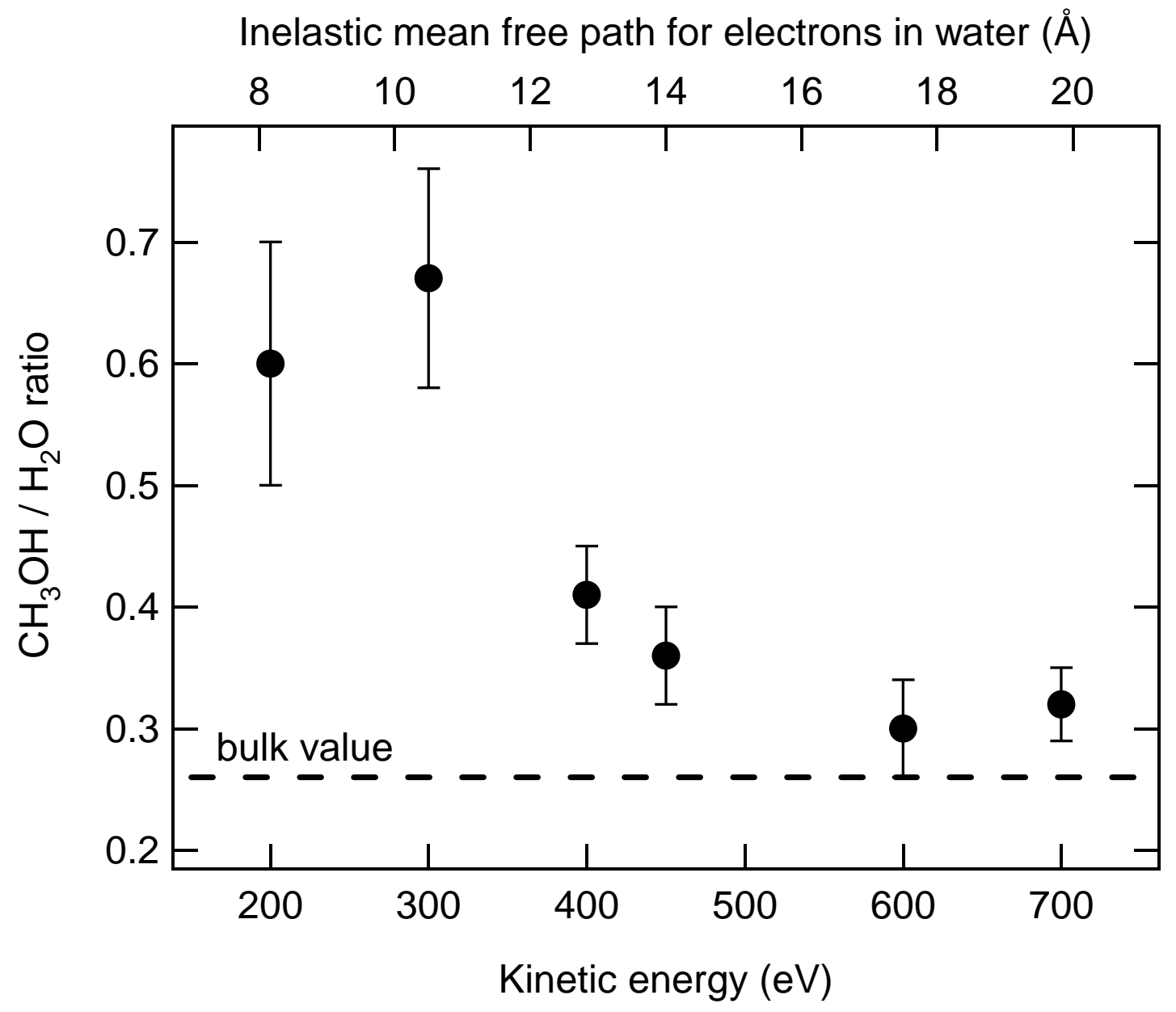

D.E. Starr, E.K. Wong, D.R. Worsnop, K.R. Wilson, H. Bluhm 